\title{
ANOTACIONES SOBRE LA CIENCIA Y LA FE A LA LUZ DE LA EPISTEMOLOGÍA ACTUAL
}

\author{
LEOPOLDO JOSÉ PRIETO LÓPEZ \\ Universidad Eclesiástica San Dámaso (Madrid)
}

\begin{abstract}
RESUMEN: La ciencia ha sido utilizada frecuentemente como instrumento de refutación de la religión. El intento más decidido en esta dirección lo ha realizado el positivismo, que identificando ciencia y verdad, declaraba falsas las afirmaciones de la religión. El neopositivismo, posteriormente, apelando al principio de verificación empírica, en lugar de declarar falsas las posiciones de la religión, las considera carentes de sentido. La actual epistemología, sin embargo, ha criticado el uso que se hace de dicho principio. Así pues, el staus quaestionis actual sobre la relación ente ciencia y fe se asienta sobre dos fundamentos: si, de un lado, las diferencias de objeto, método y fines entre ciencia y religión hacen lógicamente imposible una refutación de la religión por la ciencia, de otro lado, la misma ciencia admite la existencia de un espacio abierto para garantizar una certeza (fundada en la fe) que ella misma no es capaz de proporcionar.
\end{abstract}

PALABRAS CLAVE: ciencia, religión; positivismo, neopositivismo, principio de verificación experimental.

\section{Notes on science and faith in light of the current epistemology}

ABSTRACT: Science has frequently been used as a tool for refute religion. The most serious attempt in this direction has been made by positivism, identiying science and truth and declaring false the claims of religion. Neopositivism subsequently invoking the principle of empirical verification, instead of declaring false religion, considered it meaningless. The current epistemology, however, criticized the use made of this principle. Thus, the current status quaestionis on the relationship between science and faith rests on two foundations: on one hand, whether the differences of method and purpose between science and religion do logically impossible a refutation of religion by science; on the other hand, science itself admits the existence of an open space to ensure certainty (based on faith) that itself is unable to provide.

KEY WORDS: science, religion; positivism, neopositivism, experimental verification principle.

1. LA CIENCIA HA SIDO UTILIZADA COMO INSTRUMENTO DE REFUTACIÓN

Y LUCHA CONTRA LA RELIGIÓN

No es una verdad nueva que la ciencia ha sido utilizada por largo espacio de tiempo por el ateísmo como instrumento de refutación de la religión y en general de la visión teísta del mundo y del hombre. El viejo positivismo se distinguió de un modo particular en este uso en clave atea de la ciencia. Sea contraponiendo las teorías evolucionistas a la perspectiva creacionista, sea proponiendo una psicología naturalista como doctrina adecuada y suficiente sobre las facultades superiores del hombre, el ateísmo se valía de una versión distorsionada de la ciencia como ariete en la lucha contra la religión. Los nombres de Carl Vogt, Jacob Moleschott, Ludwig Büchner y Ernst Haeckel, de los que hace unos años se ha publicado su intercambio epistolar, son suficientemente representativos a este respecto. En cualquier caso hay que decir que el contraste entre ciencia y fe no deriva de una oposición intrínseca entre ambas, sino que más bien expresa la forma moderna, particularmente intensa con el positivismo, y quizás más típica de la 
lucha contra la religión. Como dice Agazzi, «esta lucha ha atravesado fases diversas en las distintas épocas históricas y los eternos adversarios de la religión, que siempre han existido, han creído poder encontrar en las diversas ciencias, cuando éstas se han ido constituyendo en el seno de la cultura moderna, una especie de instrumento particularmente eficaz para proseguir su lucha por la secularización ${ }^{1}$.

Quien piensa en estos términos cree encontrar la confirmación de este prejuicio en el análisis de la historia de la ciencia. Es habitual, por ejemplo, presentar de este modo el conflicto y posterior superación de la cosmología precopernicana, que, estando basada - se dice- sobre datos de naturaleza religiosa, y no pudiendo resistir el embate de las nuevas y sofisticadas teorías científicas, tuvo que ser abandonada. El positivismo del siglo XIX, como acabamos de decir, fue el más entusiasta sostenedor del modelo del conflicto en la relación entre ciencia y religión, que presentaba la historia del espíritu humano como el irresistible progreso de la razón científica, que se libera de la superstición religiosa. En este ambiente hay que situar, sobre todo, la dos siguientes obras: History of the Conflict Between Religion and Science (New York 1874), de J.W. Draper; y A History of the Warfare of Science with Theology in Christendom (New York 1896), de A.D. White. Se podría citar también como obra característica de este estilo el libro de W.E.Lecky, History of the Rise and Influence of the Spirit of Rationalism in Europe (London 1883), aunque en esta obra la tesis del conflicto se presentaba con tonos más sobrios y moderados. En cualquier caso, el esquema empleado por esta teoría era siempre el mismo: primero, las explicaciones científicas desafían a las creencias religiosas en todos los campos; después, tras presuntuosos, pero inútiles esfuerzos de los defensores de la religión, se terminan imponiendo las doctrinas científicas.

Se puede admitir sin problemas que en algunas ocasiones se han dado episodios de mutua incomprensión entre ciencia y religión que han servido de pretexto a las clásicas lamentaciones de la ciencia contra la religión, pero la mejor prueba de que tales protestas no suponen una justificación de la inconciliabilidad de la ciencia y la religión la constituye el hecho histórico de que gran parte, e incluso la mayor parte, de los científicos a los que se debe el verdadero progreso científico de los últimos siglos han sido creyentes, a veces incluso personas muy religiosas y frecuentemente también eclesiásticos (católicos y protestantes), lo que demuestra que la fe religiosa es perfectamente compatible con la actitud intelectual que caracteriza la investigación y la práctica científicas. Las razones adoptadas por los que sostienen el planteamiento cientificista con la intención de desacreditar la religión deben ser entendidas, ante todo, como la expresión de una mentalidad polémica y la consecuencia de una actitud preconcebida que, en cuanto tal, resulta impenetrable a argumentos racionales.

2. EN REALIDAD SIEMPRE HA HABIDO TENSIONES ENTRE CULTURA PROFANA Y RELIGIÓN, DE LAS QUE LAS INCOMPRENSIONES ENTRE CIENCIA Y RELIGIÓN SON UN CAPÍTULO. DE ELLO NO SE SIGUE, SIN EMBARGO, LA CONTRADICTORIEDAD O INCONCILIABILIDAD DE AMBAS, SINO LA DIFÍCIL ARMONIZACIÓN DEL PENSAMIENTO HUMANO CON EL ÁMBITO DE LA FE

Las situaciones de conflicto entre ciencia y religión no son un hecho específicamente moderno. Son la modalidad contemporánea de una relación, nunca del todo sencilla, como es en efecto la difícil conciliación entre mensaje religioso y cultura profana.

\footnotetext{
1 Agazzi, E., Scienza e fede, Massimo, Milano 1983, 91-92.
} 
Situaciones semejantes las ha vivido la Iglesia siempre que se hizo necesario elegir si servirse o no de la cultura clásica (greco-latina en concreto) para expresar su mensaje de salvación. Esta disyuntiva fue uno de los más graves aspectos afrontados por los Padres de la Iglesia, quienes decidieron aprovechar la sabiduría clásica para dar forma y expresión a la fe. Otra situación, semejante en parte, fue la creada con la recepción de las obras de Aristóteles en el siglo XIII, en un momento en el que los grandes conceptos de impronta agustiniano-platónica, forjados a lo largo de toda la Edad Media, venían a ser en buena medida modificados por la nueva filosofía del Estagirita. Los episodios de las condenas episcopales del aristotelismo (y con él, de Tomás de Aquino) tenidas lugar en París (1277) y en Oxford (1284) dejan a las claras las dificultades y reticencias a las que la nueva filosofía debió hacer frente para ser admitida. En los tiempos modernos, no siendo ya las letras y la filosofía, sino la ciencia, el factor que más incide en la mentalidad de los hombres, no es de extrañarse que sea ésta la que sostenga la confrontación con el problema de Dios y de la fe, obligándose a investigar si este problema puede conciliarse con su propio universo conceptual. Si, a pesar de todo, entre estas diversas situaciones históricas hay alguna diferencia es ésta: mientras entre cultura pagana y cristianismo el contraste se planteaba sustancialmente en el nivel de valores inspiradores o de particulares modalidades de conceptualización de la esfera religiosa, a la que en cualquier caso le era reconocida la legitimidad de una presencia a pleno título, hoy la esfera de la ciencia parece proponer un modo de concebir el mundo en el que lo sobrenatural es desplazado y obligado a no estar ya presente.

3. LA CIENCIA NUEVA INTRODUJO UN CUADRO DE REFERENCIA CONCEPTUAL SOBRE EL MUNDO EL MUNDO, EL HOMBRE Y DiOS CUALITATIVAMENTE MÁS DIFÍCIL DE ARMONIZAR CON LA FE QUE LAS PRECEDENTES SÍNTESIS TEÓRICAS. EN EL NUEVO CUADRO CONCEPTUAL, PARTICULARMENTE EN LA FORMa QUE LE CONFIRIó EL PENSAMIENTO DE DESCARTES, ANIDABAN DOS RIESGOS NO DESPRECIABLES: A) EL MATERIALISMO, COMO LA LÓGICA CONSECUENCIA DE UNO DE SUS DESARROLLOS POSIBLES (LA REDUCCIÓN DE LAS SUSTANCIAS MATERIALES A PURA EXTENSIÓN, Y EN CONSECUENCIA EN LA REDUCCIÓN DE LA FíSICA A GEOMETRÍA), Y B) EL RACIONALISMO, DEL QUE PROCEDE INEVITABLEMENTE UNA INCLINACIÓN CONTRARIA A LA FE

El cartesianismo dejaba abiertos dos problemas, no ciertamente menores, para el futuro de la relación entre ciencia y religión. De un lado, escindiendo netamente el mundo del espíritu y el mundo de las realidades materiales, especialmente en el caso del hombre, Descartes contribuía al proceso de autonomía e independencia de la investigación natural respecto de cualquier realidad o principio metafísico, favoreciendo por tanto aquella lectura integralmente mecanicista y materialista del cosmos y de la naturaleza humana que será la característica dominante de los siglos XVII y XVIII. Esta escisión justifica la reivindicación de la filosofía de Descartes tanto de los representantes del más radical espiritualismo (piénsese en Nicolás Malebranche) como los más conspicuos representantes del materialismo (como en los casos de D'Holbach, La Mettrie, Helvetius, etc.). Por otro lado, el principio metodológico cartesiano de no admitir como verdadero sino lo que aparece con toda claridad y distinción al intelecto del investigador (lo que bien entendido implicaba un nuevo concepto de verdad), dará paso de un lado al derecho del análisis subjetivo en la investigación de las cuestiones filosóficas y teológicas, y, de otro lado, dará inicio a aquel espíritu racionalista que no tardará en dirigirse, en nombre de la suprema autoridad de la razón, contra los dogmas, la tradición, y toda forma de aceptación de una esfera de verdades que caiga fuera o más allá de cuanto el 
entendimiento humano es capaz de comprender y demostrar. Por ello, a pesar de que en las Meditationes el filósofo francés había sostenido firmemente que el orden del pensamiento es superior al de la corporeidad y que el espíritu puede subsistir en el hombre sin el cuerpo, sus sucesores se referirán más bien a su De homine para afirmar que el cuerpo no tiene necesidad alguna, para ser entendido en su funcionamiento (incluido el ámbito del mundo psíquico y de la afectividad), de presuponer el pensamiento, añadiendo también que, como el sentido común atestigua, es el pensamiento, si acaso, el que parece no poder subsistir sin el cuerpo. Así, a la larga, serán el espíritu libertino, el racionalismo y el materialismo los que saquen partido del cartesianismo, mucho antes que la filosofía espiritualista y la tradición cristiana.

4. El PUNTO DE LLEGAdA DE ESTE MOVIMIENTO DE IDEAS, DESPUÉS DE RECORRER LOS MOMENTOS DEL MECANICISMO (DEL SIGLO XVII) Y DEL MATERIALISMO (DEL SIGLO XVIII), SERÁ EL POSITIVISMO, BIEN SABIDO QUE ENTRE MECANICISMO Y MATERIALISMO EXISTE UN PRECISO NEXO CONCEPTUAL E HISTÓRICO (PRECISAMENTE EL QUE RESULTA DE REINTEGRAR A LA MATERIA, COMO PROPIEDAD ESENCIAL SUYA, EL MOVIMIENTO POR MEDIO DEL CONCEPTO DE FUERZA)

En la mentalidad positivista confluían en lo esencial las aspiraciones de los movimientos intelectuales que acabamos de referir, que además, en buena medida, habían sido hechas suyas por la filosofía de la Ilustración. Ahora bien, en la mentalidad positivista anidaba una debilidad que debía conducirla finalmente a su propia crisis. Esta debilidad del positivismo consistía en la fe, sustancialmente dogmática, que profesaba en la omnipotencia de la ciencia, apoyada en un conjunto de conocimientos científicos de nivel sustancialmente divulgativo, que bien pronto se revelarían —en expresión de un conocido epistemólogo- «un poco como una filosofía de taberna», en la que con un discurso presuntuoso se consideraba poder liquidar las grandes cuestiones relativas a la espiritualidad del hombre, la esfera de los valores y la dimensión de la transcendencia recurriendo a un poco de fósforo, alguna reacción química y algún que otro vago recurso a las leyes de la mecánica, del electromagnetismo y al principio de conservación de la energía ${ }^{2}$.

5. UN NUEVO INTENTO DE DESACREDITAR LA RELIGIÓN, Y JUNTO CON ELLA TAMBIÉN LA METAFÍSICA, PERO ESTA VEZ DE UN MODO MÁS CIRCUNSTANCIADO Y ELEGANTE, FUE LLEVADO A CABO POR EL NEOPOSITIVISMO, EL CUAL EN LUGAR DE DECLARAR FALSAS Y REFUTAR LAS POSICIONES DE LA RELIGIÓN Y DE LA METAFÍSICA, COMO CREÍA PODER HACER EL VIEJO POSITIVISMO, LAS CONSIDERA CARENTES DE SENTIDO

Después del apasionado ímpetu del positivismo hacia la ciencia y de su asalto a la religión, la filosofía neopositivista ha abandonado la actitud de refutación que tendía a declarar falsa la religión y hecho suya, en cambio, una nueva actitud: la de considerar a la religión fuera del conjunto de los problemas dotados de sentido. El neopositivismo ha procedido a este propósito del siguiente modo: haciendo coincidir (dogmáticamente) el ámbito del discurso dotado de sentido con el ámbito del discurso científico resulta fácil a los filósofos pertenecientes a esta escuela partir del hecho innegable de que Dios y el

\footnotetext{
2 Cf. Agazzi, E., Scienza e fede, 106.
} 
mundo sobrenatural no forman parte del campo conceptual del conocimiento científico y concluir que, por lo tanto, dichos objetos no tienen sentido en un discurso cualquiera que tenga pretensiones cognoscitivas en general. Salta a la vista que se trata de una afirmación fundada sobre una identificación arbitraria y dogmática entre discurso científico $y$ discurso con sentido. Sin embargo, la difusión de la mentalidad científica ha hecho difícil a muchos darse cuenta de la arbitrariedad contenida en dicha identificación. En otros términos, la pretensión del neopositivismo de elevar al rango de criterio universal de significado una condición que se consideraba como criterio de significado sólo para la ciencia (es decir, que «el sentido de una proposición es el método de su verificación») ha encontrado una acogida favorable en una cultura que había hecho de la ciencia el instrumento supremo y, no pocas veces exclusivo, de todo conocimiento. Por lo tanto, todas las afirmaciones que no son susceptibles de control empírico (y, por tanto, en particular, las afirmaciones de la metafísica y las que se refieren a la esfera de la transcendencia) no requieren ni siquiera ser refutadas, toda vez que, en cuanto carentes de sentido, se liquidan por sí mismas. Según esta doctrina, se puede todavía conceder al metafísico y al creyente conservar sus convicciones a título de actitudes emotivas, pero no como formas de conocimiento cualquiera. Desafortunadamente una persuasión de este tipo ha encontrado acogida incluso entre muchos creyentes, los cuales aceptan situar su fe fuera del plano del conocimiento y de la racionalidad, radicándola únicamente en el terreno de las instancias existenciales de su interioridad. De este modo, estas personas adoptan, aunque no se den cuenta de ello, la posición de un cientificismo amalgamado con un fideísmo irracionalista.

6. EN BREVE, POSITIVISMO Y NEOPOSITIVISMO SE CARACTERIZAN POR TENER UNA MISMA ACTITUD NEGATIVA ANTE LA RELIGIÓN, PERO DIFERENTES ESTRATEGIAS

Se puede observar que el resultado antiteístico del viejo y del nuevo positivismo tienen en sustancia un idéntico fundamento, que no es sino la actitud cientificista que identifica ciencia y conocimiento verdadero. Sin embargo, este resultado es alcanzado por cada uno de estos positivismos siguiendo caminos diversos. El viejo positivismo veía esta identificación como el resultado de una progresiva ampliación de la capacidad de la ciencia, que, con ilimitado optimismo, era considerada capaz, gracias a su incesante progreso, de en un futuro no lejano poder afrontar y resolver todos los problemas humanos. Perdido este optimismo, el nuevo positivismo, de forma más cauta a la vez que sutil, pretendía alcanzar el mismo resultado, pero ahora restringiendo el campo de los problemas dotados de sentido a aquellos que la ciencia puede, o de hecho o al menos en línea de principio, afrontar y resolver. Desde el punto de vista del esfuerzo filosófico se puede afirmar que el positivismo decimonónico asumía una actitud más honesta: cuando se esforzaba, por ejemplo, en demostrar que Dios no existe, formulaba un desafío en cierto modo constructivo, puesto que trataba de refutar los argumentos metafísicos y teológicos aportando contraargumentos tomados en préstamo, más o menos eficazmente, de la ciencia. El nuevo positivismo, en cambio, pretendía el mismo resultado, pero a un precio más barato: se sentía liberado de la tarea de refutar el discurso metafísico y teológico, porque afirmaba que el problema de Dios no existe como problema cognoscitivamente dotado de sentido, aunque pueda aparecer emotiva y vivencialmente (pero no veritativamente) importante. 


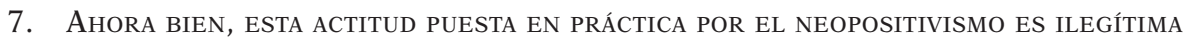

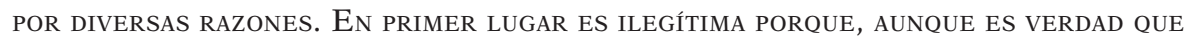
la cuestión de Dios no entra en el campo de la ciencia, ello no se debe a una PRETENDIDA INCOMPATIBILIDAD ENTRE LOS CAMPOS DE LA CIENCIA Y LA RELIGIÓN, SINO, BIEN AL CONTRARIO, A LA CONSTITUTIVA DIFERENCIA (O HETEROGENEIDAD, Y EN CONSECUENCIA, COMPLEMENTARIEDAD) TANTO DE OBJETO COMO DE MÉTODO DE ESTOS DOS ÁMBITOS DEL SABER

En primer lugar, pues, como se acaba de observar, el discurso sobre Dios no tiene cabida en la ciencia no ya porque ésta sea incompatible teóricamente con la cuestión de Dios, sino porque la ciencia no se ocupa de cuestiones que transcienden el ámbito de la observación experimental, como es el caso de Dios. Este hecho está ligado a la naturaleza intrínseca del conocimiento científico, que, en cada una de sus disciplinas, se limita a considerar solamente ciertos aspectos determinados de la realidad material y no necesita recurrir a otras hipótesis, más allá del campo conceptual circunscrito a tales aspectos, para desarrollar su trabajo. Precisamente porque la ciencia en general no se ocupa de otra cosa que de aspectos empíricamente observables de la realidad, no puede, en conformidad con su objeto y método propios, hacerse cargo de realidades como Dios, el alma espiritual, etc. Por ello, a la famosa afirmación del cirujano positivista que decía no haber encontrado nunca el alma bajo el bisturí, o a aquella jactancia del astronauta soviético que aseguraba no haber encontrado a Dios en su paseo cósmico, se debería haber replicado: si lo hubierais encontrado, lo encontrado no sería ni Dios ni el alma. Porque Dios y el alma, así como el razonamiento metafísico y religioso entienden estas realidades, no poseen carácter alguno que los haga observables o experimentables usando un bisturí o un telescopio. Ni el alma ni Dios son algo que sea razonable esperar encontrar mediante los criterios de control experimental propios de cualquier ciencia experimental. Por ello, no encontrarlos según los criterios propios del método científico no dice nada, ni a favor ni en contra, de su existencia.

8. AdemÁs, ESTA ACTITUd DEL NEOPOSITIVISMO ES ILEGítima PORQUe SIEMPRE ES POSIBLE PARA UN HOMBRE PLANTEARSE CUESTIONES QUE EXCEDEN O TRANSCIENDEN EL ÁMBITO DE LA CIENCIA, INDAGANDO ASÍ, BAJO LA PERSPECTIVA DE LA TOTALIDAD MÁS ALLÁ DE AQUELLOS ASPECTOS PARCIALES DE LOS QUE SE CONSTITUYE EL OBJETO DE LA CIENCIA

O de otro modo, el científico conserva siempre intacto el derecho a plantearse preguntas más allá del ámbito de la ciencia que cultiva, buscando una interpretación en clave de totalidad (en amplitud y profundidad) de aquellos fenómenos o aspectos parciales de la realidad material que su ciencia toma en consideración. Ahora, es claro que tratando de responder a tales preguntas ulteriores, se adentra en un modo de conocer que no es científico (en el sentido de ciencia experimental), sino filosófico; y aunque él sea un científico, en cuanto hombre que indaga y reflexiona sobre el significado de la vida y del propio mundo de la naturaleza, podrá alcanzar un conocimiento filosófico y religioso de aquellos ámbitos de la realidad que transcienden el campo de la ciencia. 
9. Llegados a ESTE PUNTO ES NECESARIO RECUSAR EL PRINCIPIO DE VERIFICACIÓN, POR CUYO MEDIO EL NEOPOSITIVISMO PRETENDÍA REDUCIR EL CONOCIMIENTO AL CONOCIMIENTO CIENTÍFICO, MÁS ALLÁ DEL CUAL CUALQUIER AFIRMACIÓN CARECERÍA DE SENTIDO

La objeción neopositivista consiste en decir que este ir más allá de la ciencia caería bajo la situación o estado de la carencia de sentido, o al menos de sentido cognoscitivo. El neopositivismo concede que sólo en cuanto hombre, con su conjunto de problemas existenciales y emotivos, el científico puede adentrarse en un discurso sobre lo sobrenatural, lo cual no tiene sin embargo sentido cognoscitivo precisable alguno. Pero esta afirmación neopositivista deriva directamente de un modo muy particular y estrecho de definir el significado, tal como es codificado en el famoso principio de verificación, según el cual «el sentido de una proposición es el método de su verificación experimental».

\section{UNA VALORACión CRÍTICA DEL PRINCIPIO DE VERIFICACIÓN ES, PUES, OBLIGADA}

Un juicio crítico sobre el principio de verificación comporta, pues, dos órdenes diversos de consideración: en primer lugar, se trata de ver si es legítima la extrapolación de un criterio de significado introducido para la ciencia empírica al rango de criterio universal de significado. En segundo lugar, se puede plantear la pregunta de si para la propia ciencia empírica está justificada la adopción de semejante criterio de significado.

\section{PRIMERA CRÍtiCa: CON EL PRINCIPIO DE VERIFICACIÓN SE INCURRE DOGMÁTICAMENTE EN UNA INDEBIDA EXTRAPOLACIÓN, COMO ES EL EXTENDER Y UNIVERSALIZAR UN CRITERIO METODOLÓGICO PROPIO DEL SABER EXPERIMENTAL A CUALQUIER FORMA DE CONOCIMIENTO}

Sobre este primer punto, la crítica filosófica se puso en marcha desde el inicio mismo de la formulación de este principio por parte tanto de pensadores ajenos a la escuela neopositivista, como de otros relacionados con ella. Estos autores llamaron la atención, en sustancia, sobre el carácter dogmático de la universalización del criterio de significado, porque éste era válido únicamente para las ciencias empíricas, y pusieron además de manifiesto que dicha universalización comportaba además una forma de contradicción interna. En efecto, si toda proposición debe ser empíricamente verificable, a fin de poseer un significado, se tiene todo el derecho del mundo de observar que el propio principio de verificación no es empíricamente verificable, y que, por tanto, es él mismo un enunciado carente de sentido.

12. Segunda CRítica: PERo ES QUe, ADEMÁs, Ni SiQuiera la CiENCIA EMPírica ESTÁ EN DISPOSICIÓN DE CUMPLIR LOS REQUISITOS DE UN CRITERIO TAL DE VERIFICACIÓN

El hecho quizás más importante es que la misma historia del desarrollo del movimiento neopositivista ha sacado a la luz la debilidad de este criterio de verificación. Ya se ha dicho que ni siquiera para la ciencia experimental se puede adoptar como criterio 
de significación el principio de verificación experimental, porque nos veríamos en la obligación de eliminar del ámbito de la ciencia experimental no solamente todas las construcciones teóricas, sino incluso las simples afirmaciones universales de las más elementales leyes físicas. Los mismos neopositivistas se vieron obligados a practicar sucesivas liberalizaciones del primitivo criterio de significado, con el fin de no tener que declarar como carente de sentido todos los componentes teóricos de las ciencias.

\section{UNA MEDIACIÓN METAEMPÍRICA (ES DECIR, FILOSÓFICA) ES, PUES, IMPRESCINDIBLE PARA LA CIENCIA EMPÍRICA}

Expresando una vez más este resultado en términos filosóficos, y no simplemente en términos de análisis metodológico, podemos decir que para las mismas ciencias empíricas se ha revelado indispensable admitir una mediación metaempírica, o sea dar nuevamente valor a aquel instrumento intelectual que es la clave para afrontar de modo cognoscitivo la mediación metafísica y, con ella, el discurso racional sobre las realidades que exceden el campo de la experiencia. Podríamos decir, por tanto, que la misma reflexión metodológica sobre la ciencia ha mostrado la imposibilidad de cerrar el espacio conceptual de la metafísica, aunque obviamente no es tarea de la ciencia estudiar si dicho espacio se puede en realidad llenar de un modo no exclusivamente postulatorio. Con ello se está autorizado para afirmar que cuando, en nombre de la visión científica del mundo, se considera válido excluir la legitimidad de una indagación racional sobre lo que transciende la experiencia, se hace una afirmación que no es sostenible precisamente según los más maduros resultados de la investigación sobre las condiciones epistemológicas de la ciencia.

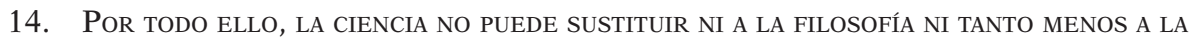
RELIGIÓN. CiENCIA Y RELIGIÓN DIFIEREN SUSTANCIALMENTE SEA EN SUS RESPECTIVOS OBJETOS COMO EN SUS FINES

Se puede decir, por tanto, que la recíproca insustituibilidad de ciencia y religión resulta del hecho de que ambas disciplinas, por así decir, se mueven en planos esencialmente diferentes, por lo que se refiere tanto al tipo de problemáticas afrontadas como a las finalidades pragmáticas que caracterizan a cada una de ellas. Este segundo aspecto, o sea la diversidad de fines, era ya claro desde hace mucho tiempo, en el sentido de que los objetivos de la ciencia (conocimiento del mundo natural y posibilidad de dominarlo en uso de dicho conocimiento) siempre ha sido claro que son diferentes de los de la religión, que pretende dar un sentido a la vida del hombre apelando a la dimensión de la trascendencia que encuentra en Dios su fundamento. En cambio, sólo en una época relativamente reciente las investigaciones filosóficas sobre la ciencia han clarificado adecuadamente cómo el tipo de saber que la caracteriza tiene una significación esencialmente diferente de cualquier otro saber que se proponga como respuesta a la problemática religiosa del mundo y de la vida. La moderna epistemología, es decir la filosofía de la ciencia, ha clarificado en efecto que el saber científico es in- trínsecamente no absoluto (es no incontrovertible) y no autofundado. 


\section{La Naturaleza y LOS Tipos DEL SABER CiENTíFico}

Las ciencias pueden subdividirse en dos grandes sectores: el de las ciencias de tipo formal, en el que se encuentran las diversas ramas de las matemáticas, y el de las ciencias de tipo experimental, integrado en general por las ciencias de la naturaleza, además de aquellas ciencias que estudian los productos de la actividad humana y al hombre mismo en sus dimensiones naturales.

Acerca de las ciencias formales, se reconoce actualmente que estas tienen la naturaleza intrínseca de sistemas hipotético-deductivos, o lo que es igual, de complejos organizados de enunciados lingüísticos, que, en rigor, no pueden ser considerados ni verdaderos ni falsos. En efecto, no se estima que dichos enunciados describan las propiedades de un sector cualquiera de la realidad, sino que pueden recibir interpretaciones capaces de verificarlos o contradecirlos a propósito de un determinado sistema de objetos. La característica peculiar de tales constructos formales es la de estar organizados de tal modo que todas las proposiciones de un cierto sistema resulten depender lógicamente (es decir, sean lógicamente deducibles) de un pequeño número de axiomas iniciales. En realidad el único requisito decisivo exigido a tales sistemas la coherencia lógica.

De este modo, el carácter no absoluto del conocimiento matemático resulta ligado al hecho de que toda proposición matemática no tiene nunca una razón en sí misma, sino que su afirmación depende del hecho de que se han asumido ciertos axiomas más bien que otros, mientras que, de otro lado, no existe ninguna razón que obligue a escoger ciertos axiomas. Un tiempo atrás se pensaba, por el contrario, que su elección era impuesta por su evidencia, pero por razones que no es cuestión de discutir aquí se ha visto la necesidad de abandonar esta convicción. Aunque no es absoluto, un sistema axiomático puede, sin embargo, resultar fundado cuando sea posible demostrar que no es contradictorio, o sea que de él no resultan contradicciones cuando se deducen consecuencias lógicas de sus axiomas. Pero, ¿cómo determinar si un sistema es o no contradictorio? Las investigaciones de la moderna lógica matemática han aclarado que ningún sistema formal es capaz de formular los medios suficientes para probar su carácter no contradictorio. Este carácter puede ser establecido (cuando se puede) únicamente recurriendo a consideraciones externas al sistema. En otros términos, tal cosa equivale a decir que ningún sistema formal es capaz de asegurar su proprio carácter de fundado.

En cuanto a las ciencias experimentales, éstas se proponen ofrecer descripciones verdaderas de los fenómenos que estudian, pero igualmente la investigación epistemológica ha concluido que éstas, en realidad, pueden al máximo construir teorías sobre tales fenómenos, las cuales, aún alcanzando un elevado grado de plausibilidad cuando son confirmadas por la experiencia, no dejan por ello de ser siempre provisionales en línea de principio. En efecto, no sólo puede ocurrir siempre que la experiencia ofrezca en un cierto momento hechos nuevos que las teorías existentes no pueden explicar, y entonces deben ceder el puesto a nuevas teorías más adecuadas, sino que vale también en línea general que una teoría obtenga confirmación sólo a partir de la verdad de ciertas consecuencias que se recaban de ella, siendo así que la lógica enseña que la verdad de las conclusiones es condición necesaria, pero no suficiente, para establecer la verdad de las premisas de las que estas son recabadas. Por tanto, aunque una teoría no haya sido desmentida por la experiencia, nunca es por este sólo hecho verdadera. Tampoco las ciencias experimentales son, pues, caracterizadas por un carácter no absoluto de sus afirmaciones. Por otro lado, tampoco las ciencias experimentales son nunca autofundadas. En efecto, utilizan un gran número de principios lógicos, de instrumentos deductivos, de cánones metodológicos, que son tenidos por válidos, pero cuya justificación no tiene lugar en el seno mismo de la ciencia que los emplea. 
16. De ello se sigue due el Saber científico es un Saber no abSoluto, no autofundado y NO INDISCUTIBLEMENTE CIERTO

En conclusión, no teniendo un valor absoluto y no poseyendo todas las razones de la propia fundamentación, la proposición científica es por esencia refutable y el saber científico se presenta por ello como un saber extremadamente riguroso (en cuanto que la admisión de sus proposiciones se realiza sobre la base de criterios metodológicos, tanto deductivos como inductivos, cuidadosos y explícitos), pero, sin embargo, no indiscutiblemente cierto. Es el caso de observar que estos resultados a los que ha llegado la moderna investigación filosófica sobre la ciencia, significan exactamente lo contrario de lo que es la convicción general de qué es la ciencia. Según la convicción popular, decir que algo está científicamente probado equivale a decir que una determinada afirmación está establecida con absoluta certeza y más allá de toda duda. Por tanto, la difusa convicción según la cual existen dos tipos de problemas: aquellos problemas sobre los que cabe alcanzar la certeza (y que están dominados por la ciencia) y aquellos otros en los que, en cambio, cada uno piensa más o menos como quiere (referidos estos a las cuestiones de fondo sobre los valores supremos y el sentido de la vida) se revela exactamente como la convicción más anticientífica que hoy se pueda sostener.

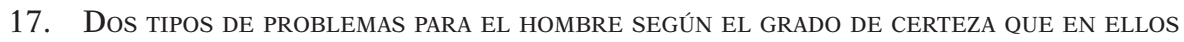
SE BUSCA: El PROBLEMA DE LAS COSAS EN GENERAL, EN EL QUE AL HOMBRE NO URGE UNA RESPUESTA INCONTROVERTIBLE, Y EL PROBLEMA DE LA PROPIA VIDA, EN EL QUE EN CAMBIO SE JUEGA TODO, Y POR TANTO LA NECESIDAD DE UNA CERTEZA ABSOLUTA ES URGENTE

La investigación científica no proporciona una certeza absoluta. Pero dicho tipo de investigación no elimina en el hombre (y en el mismo científico en cuanto hombre) la aspiración a una certeza absoluta, o por lo menos, a algunas certezas fundamentales.

Para entender bien la cuestión vale la pena subrayar una diferencia en los tipos de problemas en los que el hombre está interesado. Existen, en efecto, problemas sobre los cuales al hombre no urge una respuesta incontrovertible: entre estos están, a pesar de todas las apariencias, precisamente todos los problemas científicos. Es claro que se puede estar extremadamente interesado en saber si el teorema de Fermat, por ejemplo, es verdadero o falso, o bien en saber si el modelo en estratos del núcleo atómico es verdaderamente satisfactorio. Pero es igualmente claro que una certeza incontrovertible sobre estos problemas no es, objetivamente hablando, necesaria para dar significado a la vida y que de tal certidumbre no depende nada verdaderamente esencial para la vida humana, sino sólo para la satisfacción de la sed de conocimiento. Es, precisamente, lo que Pascal, eminente matemático y físico, a la vez que filósofo y teólogo, sugiere en sus Pensées cuando advierte que «toda la filosofía [entiéndase filosofía natural, que es como por entonces se llamaba a la ciencia experimental] no vale una hora de pena» frente a los verdaderos problemas de la vida humana, que en su opinión, son de orden moral y religioso $^{3}$. Según Pascal, tales problemas de la ciencia son diversión y en última instancia, frente a las grandes cuestiones de la vida del hombre, pérdida de tiempo.

3 Cf. Pascal, B., Pensées, 84/79 (Lafuma/Brunschvicg): «Descartes. Il faut dire en gros: cela se fait par figure et mouvement. Car cela est vrai, mais de dire quelles et composer la machine, cela est ridicule. 
Existen, en cambio, problemas sobre los que el hombre aspira necesariamente a una certeza absoluta: son aquellos en los que se juega la vida, aquellos a cuya solución está unido el sentido de la existencia, aquellos problemas de los que depende verdaderamente su destino, en el sentido de que resolverlos bien o mal puede significar el ganar o perderlo todo. Sobre estos problemas el hombre aspira a una certeza, porque, mientras puede llevar una vida dedicada a la investigación científica conducida bajo hipótesis, no puede jugarse la vida simplemente bajo hipótesis.

Sobre estos problemas últimos la ciencia, considerando todo lo afirmado hasta ahora, no puede tener respuestas y, desde luego, no constituye el tipo de saber adecuado para resolverlos, precisamente porque no ofrece la respuesta irrefutablemente cierta que para ellos es exigida. De esto se había dado cuenta el propio Wittgenstein, uno de los más importantes autores del neopositivismo, que, aún después de haber sostenido que los únicos problemas dotados de sentido son los científicos, se vio obligado a admitir que «aunque todos los problemas de la ciencia fueran resueltos, nada se habría hecho sin embargo con los verdaderos problemas del hombre» ${ }^{4}$.

\section{LA MISMA CIENCIA MUESTRA LA EXISTENCIA DE UN ESPACIO ABIERTO PARA GARANTIZAR AL HOMBRE UNA CERTEZA QUE ELLA MISMA NO ES CAPAZ DE PROPORCIONARLE}

Estamos así ante dos hechos: de un lado la existencia de problemas que requieren una solución de tipo absolutamente cierto y, del otro, la constatación de que la ciencia no puede procurar soluciones de tal tipo. Es, pues, la ciencia misma la que, en virtud de esta constatación, indica la existencia de un espacio abierto para otros tipos de investigación, o sea para tipos de investigación profundamente diversos en cuanto a finalidad, métodos, mentalidad, aspiraciones. Dentro de este espacio encuentran su lugar, con perspectivas diversas, pero con una común aspiración a una situación de certeza, la filosofía y la religión. Naturalmente, sería de un simplismo inaceptable decir que la certeza estricta, que no es dada a la ciencia, favorece en cambio a la filosofía y a la religión. Se puede decir sólo que si esta certeza puede de algún modo darse, ello será en estos niveles más bien que en el nivel de las ciencias, pero se dará también con instrumentos y métodos diversos de los de las ciencias.

19. La FE EN LA CIENCIA DEL ATEO Y DEL CIENTIFICISTA SE DEMUESTRA SIN EMBARGO UNA FE INADECUADA (EN CUANTO QUE PARA ELLOS LA RELIGIÓN MÁS QUE ELIMINADA, CAMBIA DE OBJETO) Y FIDEÍSTA (PORQUE NO ADVIERTEN LA INCAPACIDAD DE LA CIENCIA PARA RESPONDER A LAS EXIGENCIAS DE LA RELIGIÓN)

Dejando de lado ahora el problema de la filosofía, podemos decir que en la religión la certeza se da, pero se funda sobre una $f e$. Éste es, pues, el aspecto primordial e ineliminable de la religión, que no puede por ello mismo ser en modo alguno sustituida por la ciencia. Esa es también la razón de por qué el ateo sigue siendo, aunque a su pesar, religioso, en una cierta medida. También él, en efecto, se juega la existencia sobre

Car cela est inutile et incertain et pénible. Et quand cela serait vrai, nous n'estimons pas que toute la philosophie vaille une heure de peine)».

4 Wittgenstein, L., Tractatus logico-philosophicus, 6. 52. La cursiva es mía. 
algunas certezas que considera incontrovertibles y que constituyen el conjunto de ideales y valores a los cuales se adhiere y presta fe (entre los que puede tener cabida también el rechazo de la religión). También el ateo, pues, tiene su propia fe. Incluso cuando considerase que la ciencia absorbe totalmente el horizonte de los problemas humanamente dotados de sentido, haría con ello un acto de fe en la ciencia, y así dejaría de considerarla puramente como ciencia, en cuanto que le reconocería una función religiosa, a saber, precisamente la función de conferir un sentido a la existencia y de procurarle así las certezas a partir de las cuales jugarse la vida. En otros términos, ni siquiera así el ateo conseguiría eliminar el horizonte religioso, sino que se limitaría a ocuparlo con algo distinto e inadecuado, como es la ciencia, cerrando los ojos, movido de un verdadero impulso fideísta, a lo inapropiado de esta fe suya.

\section{Dos Posibles CAMinos De RELACIÓN ENTRE LA CIENCIA Y LA FE}

\subsection{La vía del método de la ciencia al objeto de la religión: contra el fideísmo}

Un encuentro entre la perspectiva científica y la búsqueda de Dios es deseable, al menos en este sentido: es importante que la lección de rigor contenida en la investigación científica no se pierda también en aquel género de investigaciones tendentes a la adquisición de certezas absolutas y en general al desarrollo de algunos contenidos cognoscitivos de la investigación sobre lo sobrenatural. En otros términos, si es verdad que es tarea esencial de la fe imprimir el sello de la certeza absoluta a los puntos fundamentales del conocimiento religioso, es también verdad que nuestra adhesión a lo divino y nuestro intento de comprender de ello algunos aspectos no deben ser puramente fideístas. Una cierta medida de rigor científico debe, pues, estar presente, por lo que concierne al método, en nuestro discurso teológico. De este modo, la ciencia puede contribuir a mejorar la calidad de nuestra investigación racional sobre los mismos problemas de la religión.

20.2. La vía del espíritu de la religión al objeto de la ciencia: contra el seco sentido científico de la naturaleza. Todavía hay espacio para la maravilla y el sentido del misterio ante al cosmos

De otra parte, así como hay un camino que va del método de la ciencia al objeto de la religión, puede haber, y de hecho hay, otro camino igualmente fecundo que va del espíritu de la religión al objeto de la ciencia. La cuestión es ésta: la investigación científica no excluye, de hecho, la posibilidad de un sentido sacral del cosmos, que, una vez aclarado su naturaleza no científica, puede convivir de un modo fecundo junto al conocimiento científico de la naturaleza, en el nivel de dación de sentido. Un tipo de consideración de este género no es, claro está, un complemento cognoscitivo de la labor de investigación científica, y tanto menos un sustitutivo de la misma. Esta visión no aumenta nuestro saber sobre el mundo, pero enriquece las dimensiones de nuestro contacto con la realidad, estimula el espíritu y nuestra capacidad de dar significado a la vida y al mundo.

No es necesario, en efecto, aceptar la idea de que la ciencia, por el hecho de haber proporcionado al hombre el instrumento más adecuado para escrutar la naturaleza y conocerla en sus secretos, deba ser por ello mismo considerada como el único ojo con el que el hombre moderno está legitimado para mirarla. El espectáculo de un ocaso o 
de un paisaje, la grandeza del cielo estrellado sobre mí, en expresión de Kant, la belleza de una flor, etc., conservan intacto todo el derecho de hablar, por ejemplo, a nuestro sentido estético, incluso después de que la ciencia nos haya dicho en qué consisten o cómo están hechos estos fenómenos. Por eso mismo estos hechos tienen el derecho de seguir hablando a nuestro sentido religioso. La ciencia los constata, describe, e intenta explicar, pero su sentido se le escapa. Considerar el cosmos bajo perspectivas distintas de las del puro interés científico no es en modo alguno concesión a una mentalidad retrógrada, precisamente porque no se trata de algo que se pone como alternativa o en contraste con la posibilidad de una investigación científica, sino porque es simplemente una capacidad de ver más, es decir, de buscar y encontrar un sentido a los hechos que la ciencia ni siquiera atisba.

\section{Actualidad DE LA FE CRISTiana}

La actual crisis del cientificismo y la constatación de las serias dificultades del hombre de dominar el progreso científico-tecnológico son, pues, condiciones favorables para que la cultura actual vuelva a poner sobre la mesa los problemas de los fines, del por qué, del sentido de la vida y de la historia... y de la orientación a dar a la misma ciencia, que es una cuestión no científica. Pero todas estas cuestiones, como puede verse, no son sino algunos componentes fundamentales del problema de Dios, sin querer naturalmente reducir tal problema a estos únicos componentes. En cualquier caso, hoy tal problema se presenta fundamentalmente en estas formas, porque el problema de Dios es esencialmente un problema de orientación de fondo, de conversión, de salvación, de elecciones radicales, de determinación de los fines por los que merece la pena empeñarla vida, de referencia a sentidos globales, no solamente para la vida individual, sino también para la vida en su conjunto, es decir, para la sociedad y la historia. Se recupera de este modo también aquella dimensión escatológica que es inherente al problema de Dios desde sus orígenes. Todas estas indicaciones emergen ahora a la superficie y no hay necesidad de excavar en profundidad para conseguir descubrirlas.

Por eso se puede considerar que en la batalla, por así decir, por el alma de la civilización contemporánea vencerá quien sepa dar una respuesta adecuada al problema de Dios, venga esta respuesta de las religiones históricas o venga de cualquier otra parte. No está dicho que tengan que ser los cristianos los que sepan dar la mejor respuesta. Pero es claro, por otro lado, que los cristianos se encuentran objetivamente en la mejor condición para darla, en cuanto que para ellos no se trata solamente de interpretar exigencias implícitas. Los cristianos han sido formados y educados en una tradición, teológicamente fundamentada como ninguna otra, que concede la prioridad a aquellas dimensiones transcendentes que hoy se ven emerger confusamente (de donde el riesgo de superstición y fanatismo) y que son, por así decir, descubiertas fatigosamente por la humanidad en camino.

Desafortunadamente, se tiene la impresión de que pocos perciben el problema desde esta óptica. En muchos cristianos es todavía dominante la impresión de estar bajo ataque, de tener que defender una trinchera, de encontrarse en la obligación de mantener alta una bandera contra la que creen que el mundo actual combate, mientras que en realidad son llamados, aunque frecuentemente de un modo inconsciente, precisamente por quienes esperan aquel tipo de respuesta que algunos cristianos creen sea contestada por esta época. Dicho brevemente, este podría ser el sentido de una reflexión no superficial, 
sino que sepa ir más allá de los signos externos de una aparente polémica, para descubrir el sentido de una posibilidad real de encuentro entre Dios y el mundo contemporáneo.

De otro lado, si el cristiano está de verdad convencido de que Dios es el bien absoluto y el valor más alto, no puede considerar que la civilización contemporánea pueda prescindir de él. Quien considere que la tarea fundamental del cristiano sea sustancialmente la de defender la realidad de Dios muestra ya estar equivocado y no creer en serio en el carácter absoluto de este valor, en virtud del cual Dios es buscado también de parte de aquellos que dicen rechazarlo. Si los cristianos no tienen ésta como su primera convicción, son entonces ellos los que tienen que recuperar el sentido de Dios. Descubrir las facciones que el rostro de Dios ha ido asumiendo en la historia de nuestros días, que quizás no son ya aquellos a los que estábamos tradicionalmente acostumbrados, puede ser una tarea que conlleve una cierta dificultad, pero ello no debe sorprendernos, porque Dios siembra en los surcos de la historia y no se revela sólo en los libros, incluso aún siendo estos libros sagrados.

Hoy la historia ha asumido un aspecto particular. Es una historia en la que la mentalidad científica domina la cultura. Quizás mañana tenga otro aspecto, como también otro fue su aspecto en el pasado. Precisamente por ello, proponer a Dios significa hoy, en buena medida, sugerir respuestas a aquellas preguntas que la ciencia ha hecho más acuciantes, reconociendo al mismo tiempo la incapacidad de la ciencia de darles una respuesta capaz de sustituir a las preguntas que proceden del reconocimiento y de la llamada del horizonte de la transcendencia.

Facultad de filosofía de la Universidad

LEOPOLdo PRIETo López

Eclesiástica San Dámaso, Madrid

lprieto7@gmail.com

[Artículo aprobado para publicación en diciembre de 2014] 